\section{Propiedades psicométricas de la escala APGAR-familiar en personas mayores residentes en zonas rurales multiétnicas chilenas}

\author{
CECILIA MAYORGA-MUÑOZ ${ }^{1, \mathrm{a}, \text { LORENA GALLARDO-PERALTA }}{ }^{2, \mathrm{~b}}$, \\ JOSÉ LUIS GALVEZ-NIETO ${ }^{1, \mathrm{c}}$
}

\section{Psychometric properties of APGAR-family scale in a multiethnic sample of Chilean older people}

Background: The APGAR-family scale is used for the assessment of family functioning in primary health care. Aim: To examine the psychometric properties of the family functioning scale APGAR-family, in a multi-ethnic sample of older Chilean adults. Material and Methods: The scale was applied to 800 participants aged more than 60 years, residing in rural areas of the regions of Arica and Parinacota and La Araucania. Results: The Cronbach's alpha obtained was 0,992 for the scale composed of five items. The confirmatory factor analysis determined a unifactorial model, whose goodness of fit indices were satisfactory. Namely, weighted least square mean and variance adjusted $-\chi^{2}$ was 20.097 ( $p<0.01$ ), comparative fit index was 0.997, Tucker-Lewis index was 0.995 and Root Mean Square Error of Approximation was 0.079 (90\% confidence intervals 0.049-0.091). Conclusions: The APGAR-family is a reliable and appropriate instrument to be applied in older people in Chile, specifically in elderly people residing in rural areas who belong to the Aymara and Mapuche indigenous communities.

(Rev Med Chile 2019; 147: 1283-1290)

Key words: Aging; Family Health; Psychometrics; Surveys and Questionnaires; Indigenous Peoples.

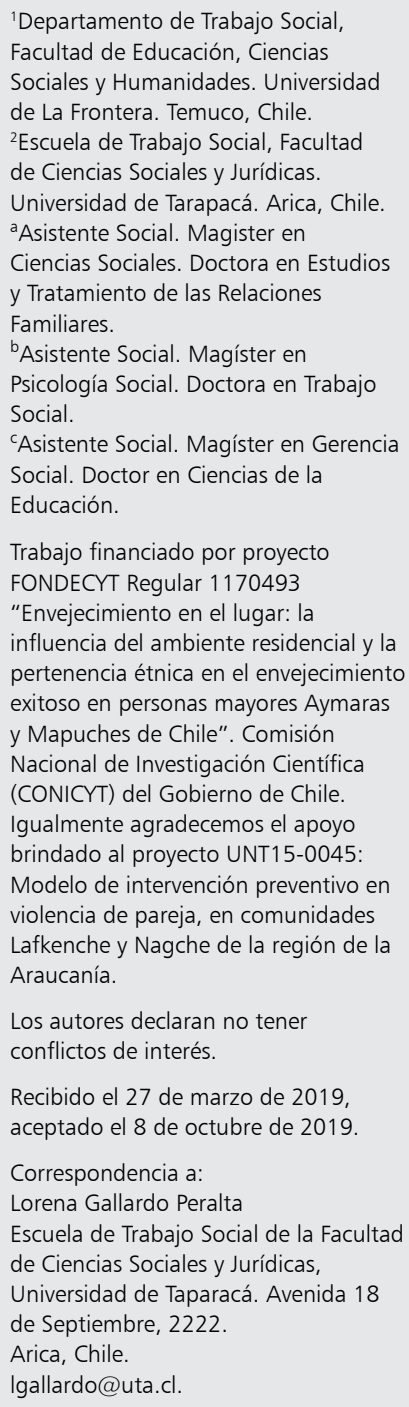

Los autores declaran no tener conflictos de interés.

Recibido el 27 de marzo de 2019, aceptado el 8 de octubre de 2019 .

Correspondencia a:

Lorena Gallardo Peralta

Escuela de Trabajo Social de la Facultad

de Ciencias Sociales y Jurídicas,

Universidad de Taparacá. Avenida 18

de Septiembre, 2222.

Arica, Chile.

lgallardo@uta.cl.

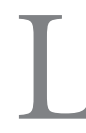

a familia ocupa un rol trascendental en el bienestar de las personas mayores ${ }^{1-4}$, teniendo en cuenta que su función se centra en dar apoyo y soporte social en circunstancias complejas propias de la vejez, tales como dependencia física, cognitiva e incluso económica. Pero, además, numerosos estudios confirman la relación directa entre el funcionamiento familiar y el bienestar en las personas mayores, ya sea en la salud mental ${ }^{5}$, en la prevención de los sentimientos de soledad ${ }^{6}$ o en el incremento de la calidad de vida ${ }^{7}$. Incluso, el funcionamiento familiar tendría una incidencia positiva en la carga de los cuidadores de los mayo$\mathrm{res}^{8} \mathrm{y}$, por el contrario, las dificultades en el funcionamiento de la familia afectan la salud integral del adulto mayor y de la familia en su conjunto?

La funcionalidad familiar es una categoría compleja de definir al incluir aspectos subjetivos y culturalmente diversos. Es por ello que teóricamente se han propuesto distintos modelos para determinar la funcionalidad de una familia ${ }^{10-12}$. La propuesta que nos ocupa en este trabajo, la de Smilkstein ${ }^{13}$, parte de la premisa que una familia es funcional en la medida que sus miembros per- 
ciban cuidado y apoyo por parte de esta. Además, el autor operacionaliza la funcionalidad familiar en cinco áreas: adaptación, participación o asociación, desarrollo o crecimiento, afecto y resolución o recursos ${ }^{14,15}$.

En el caso específico de la funcionalidad familiar en la etapa de la vejez, nos referimos a la capacidad del entorno familiar de ser flexible y adaptable a las diversas necesidades de las personas mayores. En otras palabras, el entorno familiar es una red o soporte de apoyo en la vejez, porque propicia las transacciones de ayuda y cuidado de sus miembros ${ }^{1,16}$. En esta línea, se observa que aquellas familias que dan apoyo y afecto se adaptan a las nuevas circunstancias y resuelven problemas propios de la vejez y suelen propiciar el bienestar en las personas mayores ${ }^{17,18}$. De manera específica, la funcionalidad familiar influye positivamente en el bienestar subjetivo de sus miembros, que implicaría sentirse feliz, saludable y próspero ${ }^{19}$. Sin embargo, en los últimos años han aumentado los problemas sociales vinculados a la soledad en las personas mayores ${ }^{20}$, diversas investigaciones afirman que la soledad se relaciona con la experiencia de no tener o tener una insuficiente red de relaciones que limitan la proximidad con otros y la plena integración social ${ }^{20,21}$. Frente a lo cual, la soledad se vincularía con disfuncionalidad familiar, es decir, una familia que no logra contener emocionalmente a sus miembros genera un sentimiento de discrepancia entre las relaciones sociales que desean y las que poseen realmente ${ }^{22}$.

En este trabajo nos centraremos en la validación de la escala APGAR-familiar, siendo uno de los instrumentos más utilizados en la evaluación familiar ${ }^{15}$. Su amplio uso en la valoración de las familias se debe a sus múltiples fortalezas, tales como ser una escala breve y de fácil aplicación. Además, las propiedades psicométricas del APGAR-familiar han sido adecuadas en diversas poblaciones. En este sentido, se confirma que sus cinco ítems cargan a un factor y la fiabilidad, alfa de Cronbach, suele ser entre 0,71 y $0,83^{15,23,24}$.

Esta escala ha sido validada en población adulta $^{25,26}$ y en personas mayores ${ }^{27}$. Igualmente, ha sido ampliamente utilizada en población hispana mayor en España ${ }^{2,28}$, en México ${ }^{3,9}$, en Paraguay ${ }^{29}$, en Colombia ${ }^{30}$, en Perú $^{31}$ y en Chile ${ }^{14,32,33}$, pero dichos estudios no informan de sus propiedades psicométricas y de su proceso de validación. Es necesario contar con una validación de esta escala en personas mayores y, especialmente, en personas indígenas, teniendo en cuenta que el proceso de validación garantiza la calidad de la medición. En este sentido, el objetivo de este estudio es: a) evaluar las propiedades psicométricas de fiabilidad y validez de la escala de percepción del funcionamiento familiar de Smilktein (APGAR) en una muestra multiétnica de personas mayores en Chile; b) evaluar la validez de constructo y validez emergente a través de la utilización de la Escala de soledad $^{34} \mathrm{y}$ el Índice de bienestar personal ${ }^{35}$.

\section{Método}

\section{Participantes}

Participaron en este estudio 800 personas mayores que viven en las regiones de Arica y Parinacota y La Araucanía. Se trata de un muestreo no probabilístico y por disponibilidad y los criterios de selección de la muestra fueron ser un persona mayor de 60 años, sin deterioro cognitivo y con residencia en zonas rurales.

Las características demográficas generales se presentan en la Tabla 1, destacando el hecho de que se trata de una muestra multiétnica, que se distribuye de la siguiente manera: 231 no indígenas, 201 aymaras y 368 mapuches.

\section{Instrumentos}

Funcionamiento familiar. APGAR-familiar de Smilkstein ${ }^{13}$. Evalúa la percepción del funcionamiento familiar mediante la exploración de la satisfacción del entrevistado respecto a sus relaciones familiares. Consta de cinco ítems tipo Likert $(0=$ casi nunca, $1=$ algunas veces y $2=$ casi siempre). Los puntos de corte son los siguientes: familia funcional: 7-10 puntos; familia con disfunción leve: 4-6 puntos; y familia disfuncional grave: $0-3$ puntos.

Soledad. Escala de soledad ${ }^{34}$. Evalúa la percepción subjetiva individual de la participación social o el aislamiento en población mayor. La versión abreviada de la escala está compuesta de dos dimensiones: soledad emocional y soledad social, con seis ítems ${ }^{36}$. En este estudio solo utilizaremos la dimensión de soledad emocional.

Bienestar subjetivo ${ }^{35}$. Indice de bienestar personal (PWI). Evalúa el bienestar o satisfacción global en siete dominios. El instrumento posee una escala de respuesta tipo Likert, cuyo rango oscila 
Tabla 1. Principales características de la muestra

\begin{tabular}{|llr|}
\hline Variables & Categorías & n (\%) \\
Sexo & Mujer & $393(49 \%)$ \\
& Hombre & $407(51 \%)$ \\
Edad & $60-69$ años & $341(43 \%)$ \\
& $70-79$ años & $311(39 \%)$ \\
& $80+$ años & $148(18 \%)$ \\
Estado & Casado/a o con pareja & $434(54 \%)$ \\
civil & Soltero/a & $124(15 \%)$ \\
& Viudo/a & $190(24 \%)$ \\
& Divorciado/a, separado/a & $52(7 \%)$ \\
Nivel de & Educación básica incompleta & $433(54 \%)$ \\
estudios & Educación básica & $245(31 \%)$ \\
& Educación media/técnico superior & $108(13 \%)$ \\
& Educación superior & $14(2 \%)$ \\
Etnicidad & Indígenas (aymara/mapuche) & $569(71 \%)$ \\
& No indígenas & $231(29 \%)$ \\
\hline
\end{tabular}

entre 0 y 10 puntos. Esta escala ha sido validada en personas mayores chilenas, incluidas personas mayores indígenas ${ }^{37}$

\section{Procedimiento}

Se realizó un estudio piloto con 20 individuos, 10 aymaras y 10 mapuches, aplicándose la escala en español disponible en el repositorio del Ministerio de Salud ${ }^{38}$ y no fue necesario realizar cambios. Posteriormente, se aplicó la escala al resto de los participantes entre los meses de agosto y noviembre de 2017. El método de aplicación fue a través de entrevista personal (cara a cara) en un espacio habilitado para realizar esta actividad. Los participantes fueron contactados a través de dos procedimientos. Cuando fue posible, el equipo de investigación hizo el primer contacto directamente y organizó una cita para realizar la entrevista. Cuando el primer contacto implicó mayores dificultades, se realizó a través de agentes sociales clave (por ejemplo, el presidente de la junta de vecinos).

Esta investigación es parte del proyecto FONDECYT Regular 1170493, por tanto el Comité de Ética de la Universidad de Tarapacá supervisó y aprobó los aspectos éticos del estudio. Todos los procedimientos realizados en estudios con participantes humanos se realizaron de acuerdo con los de la declaración de Helsinki de 1964 y la normativa establecida en el Convenio 169 de la Organización Internacional del Trabajo sobre pueblos indígenas y tribales.

\section{Análisis de datos}

Con el objetivo de entregar evidencias de validez cruzada, se seleccionaron aleatoriamente dos submuestras independientes de 400 casos. Para evaluar la equivalencia de ambas muestras, se calcularon posibles asociaciones entre la variable $\operatorname{sexo}\left(\chi^{2}[\mathrm{gl}=1]=1,806 ; \mathrm{p}=0,179\right)$ y diferencia de medias para la variable edad $(\mathrm{t}[\mathrm{gl}=798]=1,076$; $p=0,282$ ), estos resultados indican que no existen diferencias estadísticamente significativas entre ambas muestras. Con la primera muestra se realizó un análisis factorial exploratorio (AFE), utilizando la matriz de correlación policórica y el método de estimación de mínimos cuadrados no ponderados (ULS), además, se utilizó una rotación oblicua (oblimin directo), estos análisis se realizaron con el software FACTOR 10.8.04 ${ }^{39}$. Ahora bien, para evaluar la estructura factorial de la escala, es decir, su ajuste a un modelo unidimensional, se consideraron los siguientes índices: Unidimensional Congruence (UniCo), Explained Common Variance (ECV) y Mean of Item Residual Absolute Loadings (MIREAL). Para UniCo se consideró un ajuste aceptable valores mayores o iguales a 0,95 , para ECV, valores mayores o iguales a 0,85 y MIREAL, valores inferiores a $0,30^{51}$.

Con la segunda muestra se realizó un análisis factorial confirmatorio (AFC), con el software MPLUS $7.11^{40}$, se seleccionó la matriz policórica y en este caso se utilizó el método de estimación mínimos cuadrados ponderados con media y varianza ajustada (WLSMV) ${ }^{41}$. Para evaluar la calidad del modelo, se utilizaron los índices de bondad de ajuste: índice de ajuste comparativo (CFI), índice Tucker-Lewis (TLI) y el error cuadrático medio de aproximación (RMSEA). Para los primeros índices (CFI y TLI) se consideraron como un ajuste aceptable del modelo valores superiores a $0,90^{42} \mathrm{o}$ un ajuste excelente valores mayores a 0,95 , mientras que para el RMSEA se consideró como ajuste razonable valores inferiores a 0,08 , o un ajuste excelente menores a $0,06^{43}$.

Con el objetivo de evaluar las evidencias de validez de criterio, se realizó un modelo de ecuaciones estructurales, analizando las relaciones entre las puntuaciones del APGAR-familiar y soledad, esperando encontrar correlaciones altas 
Tabla 2. Estadísticos descriptivos de los ítems de la escala

\begin{tabular}{|c|c|c|c|c|c|c|c|c|}
\hline Ítems & Media & $\begin{array}{l}\text { Desviación } \\
\text { típica }\end{array}$ & Mín. & Máx. & $\begin{array}{l}\text { Asime- } \\
\text { tría }\end{array}$ & Curtosis & $\begin{array}{l}\text { Prueba } \\
\text { Kolmogorov- } \\
\text { Smirnov }\end{array}$ & p-valor \\
\hline $\begin{array}{l}\text { 1. Grado de satisfacción con la } \\
\text { ayuda que recibe de su fami- } \\
\text { lia cuando tiene un problema }\end{array}$ & 1,46 & 0,683 & 0 & 2 & $-0,879$ & $-0,435$ & 0,353 & $<0,001$ \\
\hline $\begin{array}{l}\text { 2. En la familia se conversan los } \\
\text { problemas que hay en casa }\end{array}$ & 1,34 & 0,752 & 0 & 2 & $-0,653$ & $-0,958$ & 0,321 & $<0,001$ \\
\hline $\begin{array}{l}\text { 3. Se toman las decisiones en } \\
\text { conjunto en la casa }\end{array}$ & 1,28 & 0,807 & 0 & 2 & $-0,559$ & $-1,246$ & 0,320 & $<0,001$ \\
\hline $\begin{array}{l}\text { 4. Grado de satisfacción con el } \\
\text { tiempo en que Ud. y su fami- } \\
\text { lia pasan juntos }\end{array}$ & 1,36 & 0,711 & 0 & 2 & $-0,641$ & $-0,810$ & 0,312 & $<0,001$ \\
\hline 5. Siente que su familia le quiere & 1,62 & 0,610 & 0 & 2 & $-1,354$ & 0,733 & 0,420 & $<0,001$ \\
\hline
\end{tabular}

y negativas entre ellas. Para evaluar la calidad del modelo se utilizaron los índices descritos en el párrafo anterior.

Finalmente, para evaluar las evidencias de fiabilidad del APGAR-familiar se utilizó, además del coeficiente alfa, estimadores más robustos para datos en psicología ${ }^{44}$, omega ${ }^{45}$ y greatest lower bound $(\mathrm{GLB})^{46}$.

\section{Resultados}

\section{Funcionalidad física, problemas de salud y funcionamiento familiar}

Las características de funcionalidad física (índice Barthel) y problemas de salud de la muestra: $81 \%$ era independiente/autovalente, $5 \%$ tiene dependencia escasa, $12 \%$ dependencia moderada y $2 \%$ dependencia severa. Los datos sobre enfermedades diagnosticadas indicaron que 51\% tenía hipertensión arterial, 22\% dolores articulares, incluyendo artrosis y reumatismo, 22\% dislipidemia, 21\% diabetes mellitus, $12 \%$ cataratas, $6 \%$ problemas respiratorios, $5 \%$ problemas cardiacos, $3 \%$ problemas estomacales y $3 \%$ osteoporosis.

Conforme a la funcionalidad familiar percibida, $62 \%$ de la muestra tenía una familia funcional, $23 \%$ una familia con disfunción leve y $15 \%$ una familia con disfunción grave.

\section{Análisis descriptivos y exploración de la estructura factorial}

En la Tabla 2 se presentan los estadísticos descriptivos de los ítems de la escala. Al evaluar los promedios se observa que el ítem 5 "Siente que su familia le quiere" obtuvo la puntuación media más alta (media $=1,62$, desviación típica $=0,610$ ), en contraste, el ítem 3 "Se toman las decisiones en conjunto en la casa” presentó la puntuación media más baja (media $=1,28$, desviación típica $=0,807$ ). Además, al evaluar la normalidad univariada de los ítems, mediante la prueba de Kolmogorov Smirnov, se rechazó la hipótesis nula de normalidad $(\mathrm{p}<0,001)$, por lo que se ratificó la elección de métodos de estimación robustos dado el incumplimiento de este requisito.

Posteriormente, se evaluó la adecuación de la matriz de correlaciones, al analizar el coeficiente Kaiser-Meyer-Olkin $(\mathrm{KMO}=0,875)$ y el estadístico de Bartlet $\left(\chi^{2}[\mathrm{gl}=10]=1589,1 ; \mathrm{p}<0,001\right)$ estadísticamente significativo, indicando que la matriz de datos era apropiada para implementar un AFE. El análisis paralelo sugirió la presencia de

Tabla 3. Índices de ajuste unidimensional

\begin{tabular}{|cc|}
\hline Índices & Valor \\
\hline UniCo & 0,999 \\
\hline ECV & 0,962 \\
MIREAL & 0,170 \\
\hline
\end{tabular}

Nota: UniCo = Unidimensional Congruence, un valor igual o mayor a .95 sugiere unidimensionalidad. ECV = Explained Common Variance, sugiere valores mayores o iguales a 0,85 representan estructuras esencialmente unidimensionales. MIREAL: Mean of Item Residual Absolute Loadings, sugiere que valores inferiores a 0,30 representan estructuras esencialmente unidimensionales. 
una dimensión que explicaba más varianza que la esperada en matrices aleatorias -500 matrices aleatorias-, este factor explicó 78,83\% de varianza. Además, como se aprecia en la Tabla 3, los índices UniCo, I-ECV y MIREAL sugieren que la estructura factorial del APGAR- familiar es esencialmente unidimensional.

\section{Análisis factorial confirmatorio}

Con el objetivo de presentar evidencias de validez cruzada, se procedió a confirmar la estructura de la escala utilizando un AFC con los datos de la segunda muestra $(n=400)$. Para el modelo de un factor, los índices de bondad de ajuste fueron satisfactorios WLSMV $-\chi^{2}(5)=20,097 ; \mathrm{p}<0,001$; CFI $=0,997 ;$ TLI $=0,995 ;$ RMSEA $=0,079$ (IC90\% = 0,049-0,091). Estos valores indican en general, que el modelo ajusta bien a los datos, por lo que se confirmó la estructura teórica unidimensional para el APGAR-familiar (Figura 1).

\section{Evidencias de validez de criterio externo}

Para evaluar evidencias de validez de criterio

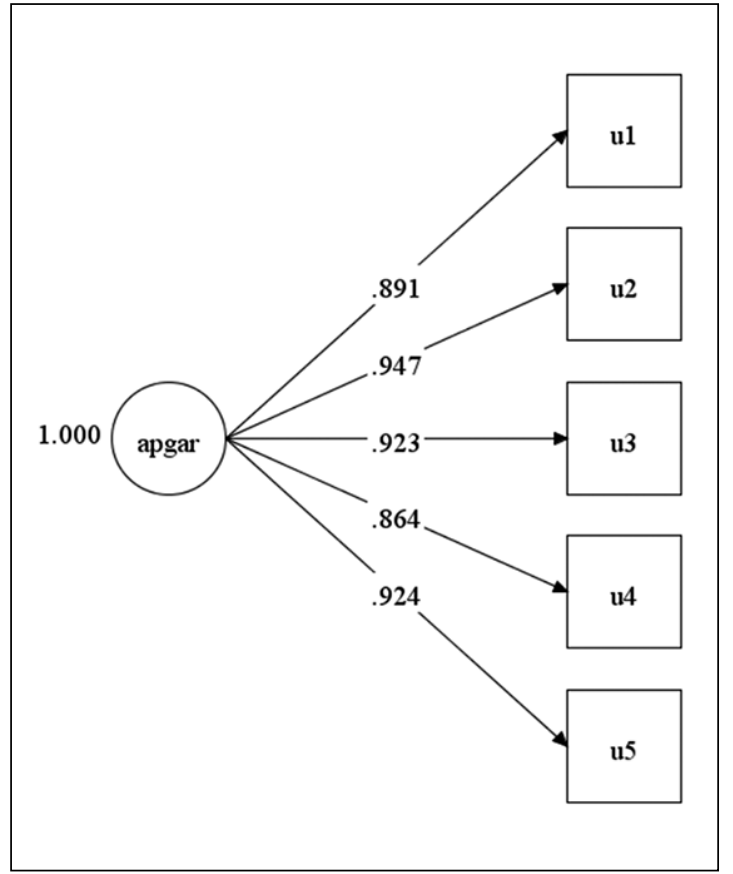

Figura 1. Modelo de un factor $(n=400)$.

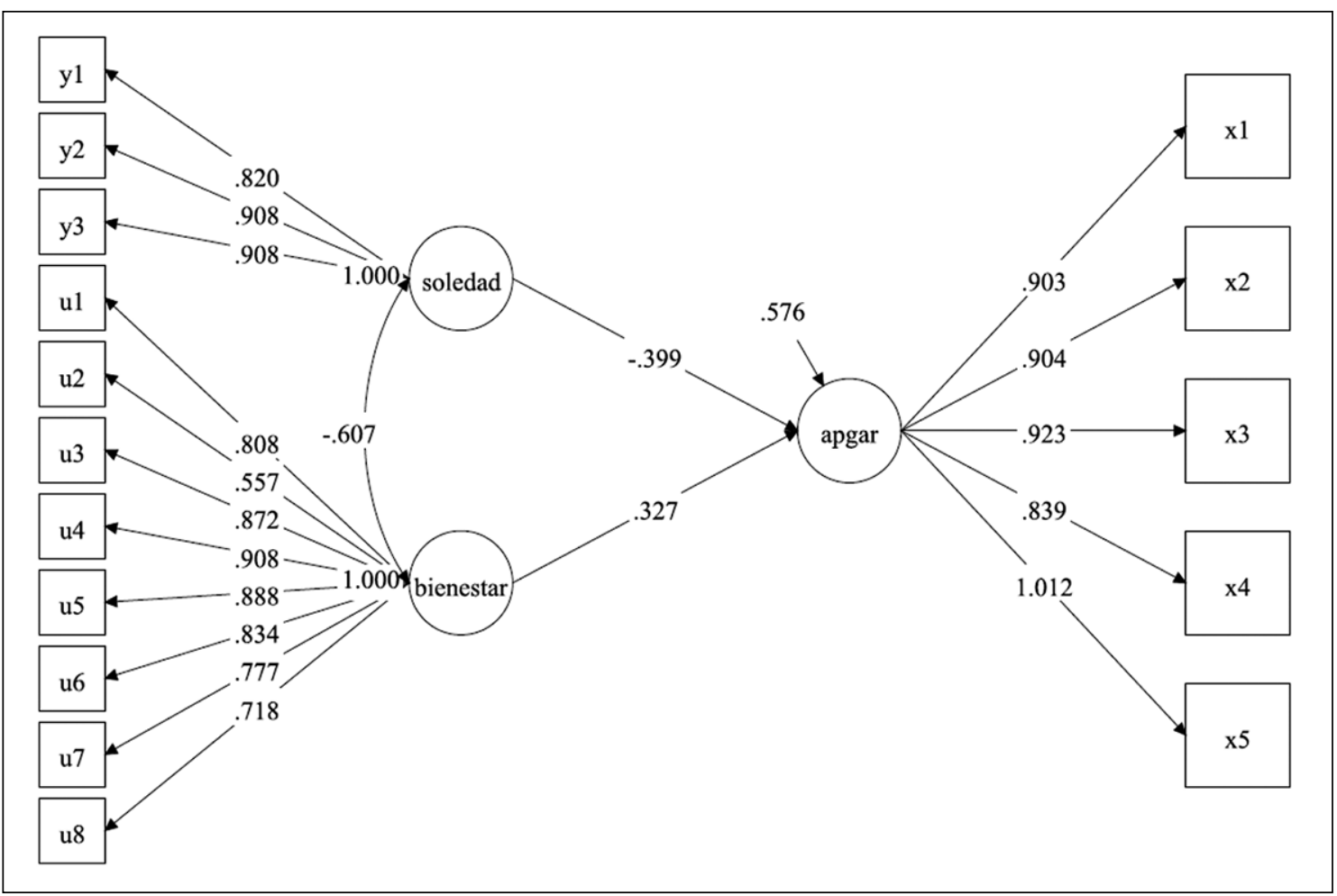

Figura 2. Modelo estructural de relaciones entre APGAR-familiar, escala de soledad y bienestar subjetivo. 
externo, se analizó un modelo de ecuaciones estructurales que relacionó el factor general del APGAR-familiar, la dimensión de soledad emocional y el factor general de bienestar subjetivo. El resultado mostró índices de bondad de ajuste satisfactorios WLSMV- $\chi^{2}(\mathrm{gl}=101)=487,933, \mathrm{CFI}=$ $0,964, \mathrm{TLI}=0,957, \mathrm{RMSEA}=0,069(0,063-0,075)$. Los resultados indicaron que el factor general de APGAR-familiar se correlacionaron de manera negativa con la dimensión de soledad y positiva con el factor general de bienestar subjetivo (Figura 2).

\section{Evidencias de fiabilidad}

Considerando las evidencias de dimensionalidad, se calcularon diversos estimadores de fiabilidad para el factor general del APGAR-familiar: Alfa $=0,992 ;$ Omega $=0,968 ; \mathrm{GLB}=0,980$. Dichos resultados proporcionaron evidencia suficiente para fundamentar que el instrumento posee niveles de fiabilidad adecuados para su aplicación en población de personas mayores chilenas.

\section{Discusión}

La escala APGAR-familiar es un instrumento ampliamente utilizado en el campo de la atención primaria en salud en Chile. Particularmente en contextos de salud familiar, ya que permite a los profesionales de la salud identificar aquellas familias con conflictos o dificultades en el cumplimiento de sus funciones ${ }^{15}$. Se debe tomar en cuenta que la funcionalidad familiar es reconocida como favorable en procesos de recuperación de la salud y en el bienestar general de las personas mayores, lo que facilitaría una mejor planificación de la atención y tratamiento en salud familiar ${ }^{46,47}$. En esta línea, en este estudio hemos evidenciado que la funcionalidad familiar se relaciona positivamente con bienestar subjetivo y negativamente con el sentimiento de soledad.

Ahora bien, dando cuenta de los objetivos propuestos, respecto a la fiabilidad, los resultados indican valores adecuados e incluso superiores a estudios previos ${ }^{15,23,24}$. Destacamos los estudios realizados en Brasil ${ }^{47}$ y en España ${ }^{27}$ con muestras transculturales de personas mayores, donde el instrumento mostró un alfa de Cronbach entre 0,80 y 0,95 , respectivamente. En lo que respecta a la validez, se analizó la estructura factorial a través del procedimiento de validez cruzada, los resultados del AFC para el modelo de un factor confirmaron la estructura teórica unidimensional del instrumento. Estudios previos han demostrado que se trata de una escala unidimensional ${ }^{23,24}$. Los valores de las cargas factoriales indican valores adecuados, con un mínimo de 0,86 y un máximo de 0,94 . Por otro lado, el análisis de validez de constructo y validez emergente permitió evidenciar la relación de la escala con las variables esperadas, demostrado una correlación moderada y en el sentido esperado con soledad $(-0,39)$ y con bienestar subjetivo $(0,32)$.

La escala APGAR-familiar tiene las ventajas de haber sido diseñada para evaluar la funcionalidad familiar con fines de intervención en salud, por tanto, es un instrumento de fácil comprensión en sus enunciados y de fácil aplicación, a diferencia de otras escalas posteriores (FACES -20 ESP, FACESIII $)^{48,49}$ que han surgido con fines más centrados en la investigación familiar. Igualmente, queremos señalar que dada la alta complejidad y heterogeneidad de las familiares actuales ${ }^{1}$, esta escala debiera ser utilizada en complemento con otros mecanismos de evaluación. En efecto, el instrumento debiera ser parte de un conjunto de instrumentos que puedan aportar información más amplia sobre las familias, no solo la funcionalidad familiar, como es el caso del SALUDFAM ${ }^{50}$.

El estudio tiene ciertas limitaciones que deben tenerse en cuenta, una de ellas es la representatividad de la muestra. Aunque se trata de una muestra representativa de las zonas rurales de las regiones de Arica y Parinacota y La Araucanía, no es una muestra nacional. Por tanto, sus alcances son limitados para el resto de la población chilena y para comunidades indígenas distintas a las investigadas. No obstante, este estudio muestra la relevancia de validar los instrumentos ampliamente utilizados en salud familiar en Chile ${ }^{38}$.

Concluyendo, los resultados de la investigación proporcionan evidencias psicométricas de la escala APGAR-familiar demostrando que es una escala fiable y adecuada para evaluar el funcionamiento familiar en personas mayores de zonas rurales e indígenas.

\section{Referencias}

1. Gallardo-Peralta LP, Barrón A, Molina MA, Schettini R. Family and community support among older Chilean 
adults: the importance of heterogeneous social support sources for quality of life. J Gerontol Soc Work 2018; 61 (6): 584-604.

2. García L, Mérida F, Mestre I, Gallego MD, Duarte AJ, Mesa RG, et al. La depresión del anciano y el papel de la dinámica familiar. Atención Primaria 2000; 25 (4): 226-9.

3. Martínez Piñón AI, Pastrana Huanaco EC, Rodríguez-Orozco AR, Gómez Alonso C. Existe correlación entre depresión y función familiar en adultos mayores que asisten a una clínica de medicina familiar. Rev Med Chile 2009; 137 (12): 1642-3.

4. Vivaldi F, Barra E. Bienestar psicológico, apoyo social percibido y percepción de salud en adultos mayores. Ter Psicol 2012; 30 (2): 23-9.

5. de Oliveira SC, Pavarini SC, Orlandi F de S, de Mendiondo MS. Family functionality: A study of Brazilian institutionalized elderly individuals. Arch Gerontol Geriatr 2014; 58 (1): 170-6.

6. Wu ZQ, Sun L, Sun YH, Zhang XJ, Tao FB, Cui GH. Correlation between loneliness and social relationship among empty nest elderly in Anhui rural area, China. Aging Ment Health 2010; 14 (1): 108-12.

7. Lu C, Yuan L, Lin W, Zhou Y, Pan S. Depression and resilience mediates the effect of family function on quality of life of the elderly. Arch Gerontol Geriatr 2017; 71: 34-42.

8. Chiou CJ, Chang HY, Chen IP, Wang HH. Social support and caregiving circumstances as predictors of caregiver burden in Taiwan. Arch Gerontol Geriatr 2009; 48 (3): 419-24.

9. Carrazco-Peña KB, Tene CE, Elizalde AM. Disfunción familiar y desnutrición en el anciano. Rev Med Inst Mex Seguro Soc 2015; 53 (1): 14-9.

10. Epstein NB, Bishop DS, Levin S. The McMaster Model of Family Functioning. J Marriage Fam Couns 1978; 4 (4): 19-31.

11. Epstein NB, Bishop DS, Baldwin LM. McMaster Model of Family Functioning: A view of the normal family. En: Walsh F, Guilford family therapy series. Normal family processes. Guilford Press. New York, USA. 1982. p. 11541.

12. Walsh F. Family resilience: Strengths forged through adversity. En: Walsh F, Normal family processes: Growing diversity and complexity. Nueva York, USA: The Guilford Press. 2012. p. 399-427.

13. Smilkstein G. The Family APGAR: A proposal for family function test and its use by physicians. J Fam Pract 1978; 6(6): 1231-9.

14. Horwitz N, Florenzano R, Ringeling I. Familia y salud familiar. Un enfoque para la atención primaria. Bol
Oficina Sanit Panam 1985; 98 (2): 144-54.

15. Suárez MA, Alcalá M. APGAR-familiar: una herramienta para detectar disfunción familiar. Rev Méd La Paz 2014; 20 (1): 53-7.

16. Chappell NL, Funk LM. Social support, Caregiving, and Aging. Can J Aging 2011; 30 (3): 355-70.

17. Gyasi R, Phillips D, Abass K. Social support networks and psychological wellbeing in community-dwelling older Ghanaian cohorts. Int Psychogeriatr 2018; 25: $1-11$.

18. Zhou G, Wang Y, Yu X, Direct and Indirect Effects of Family Functioning on Loneliness of Elderly Chinese Individuales. Curr Psychol 2018; 37 (1): 295-301.

19. Ramírez M, Lee SL. Factors associated to life satisfaction in adults over 60 years old. Polis 2012; 11 (33): 407-28.

20. López J, Díaz MP. El sentimiento de soledad en la vejez. Revista Internacional de Sociología 2018; 76 (1): e085.

21. Gené-Badia J, Ruiz-Sánchez M, Obiols-Masó N, Oliveras L, Lagarda L. Aislamiento social y soledad: ¿qué podemos hacer los equipos de atención primaria? Aten Primaria 2016; 48 (9): 604-9.

22. De Jong Gierveld, J, Keating N, Fast JE. Determinants of loneliness among older adults in Canada. Can J Aging 2015; 34(2): 125-36.

23. Castilla Cabello H, Caycho Rodríguez T, Ventrura León JL, Palomino-Barboza M, de la Cruz Valles M. Análisis factorial confirmatorio de la escala de percepción del funcionamiento familiar de Smilkstein en adolescentes peruanos. Salud \& Sociedad 2016; 6 (2): 140-53.

24. Forero Ariza LM, Avendaño Durán MC, Duarte Cubillos ZJ, Campo Arias A. Consistencia interna y análisis de factores de la escala APGAR para evaluar el funcionamiento familiar en estudiantes de básica secundaria. Rev Colomb Psiquiatr 2006; 35 (1): 23-9.

25. Bellón Saameño JA, Delgado Sánchez A, Luna del Castillo JD, Lardelli Claret P. Validez y fiabilidad del cuestionario de función familiar Apgar-familiar. Aten Primaria 1996; 18 (6): 289-96.

26. Gómez Clavelina FJ, Ponce Rosas ER. Una nueva propuesta para la interpretación de Family Apgar. Aten Fam 2010; 14 (4): 102-6.

27. García González JV, Díaz Palacios E, Salamea García A, Cabrera González D, Menéndez Caicoya A, Fernández Sánchez A, et al. Evaluación de la fiabilidad y validez de una escala de valoración social en el anciano. Aten Primaria 1999; 23 (7): 434-40.

28. Montejano AR, Ferrer RM, Clemente G, Martínez-Alzamora N, Sanjuán A, Ferrer E. Factores asociados al riesgo nutricional en adultos mayores autónomos no institucionalizados. Nutr Hosp 2014; 30 (4): 858-69. 
29. Riveros Ríos M. Valoración de factores biopsicosociales determinantes del riesgo de desnutrición en los adultos mayores en hogares de ancianos. An Fac Cienc Méd 2015; 48 (2): 17-31.

30. Díaz S, Arrieta K, Ramos K. Impacto de la Salud Oral en la Calidad de Vida de Adultos Mayores. Rev Clin Med Fam 2012; 5 (1): 9-16.

31. Varela-Pinedo L, Chávez-Jimeno H, Tello-Rodríguez T, Ortiz-Saavedra P, Gálvez-Cano M, Casas-Vasquez P, et al. Perfil clínico, funcional y sociofamiliar del adulto mayor de la comunidad en un distrito de Lima, Perú. Rev Perú Med Exp Salud Publica 2015; 32 (4): 709-16.

32. Zavala M, Vidal D, Castro M, Quiroga P, Klassen G. Funcionamiento social del adulto mayor. Cienc Enferm 2006; 12 (2): 53-62.

33. Ferrada L, Zavala M. Bienestar psicológico: adultos mayores activos a través del voluntariado. Cienc. Enferm. 2014; 20 (1): 123-30.

34. De Jong Gierveld J, Van Tilburg TG. A six-item scale for overall, emotional and social loneliness: confirmative tests on new survey data. Res Aging 2006; 28: 582-98.

35. Cummins RA, Eckersley R, Pallant J, Van Vugt J, Misajon R. Developing a national index of subjective wellbeing: The Australian Unity Wellbeing Index. Soc Indic Res 2003; 64 (2): 159-90.

36. Ayala A, Rodríguez-Blázquez C, Frades-Payo B, Forjaz MJ, Martínez-Martín P, Fernández-Mayoralas G, et al. Propiedades psicométricas del Cuestionario de Apoyo Social Funcional y de la Escala de Soledad en adultos mayores no institucionalizados en España. Gac Sanit 2012; 26 (4): 317-24.

37. Gallardo-Peralta LP, Molina MA, Schettini R. Validation of the Personal Wellbeing Index (PWI) for older Chilean adults. Int Psychogeriatr 2019; 31 (11): 1679-80.

38. Ministerio de Salud. Orientaciones para la implementación del modelo de atención integral de salud familiar y comunitaria. 2012. Disponible en: https://www.minsal. cl/portal/url/item/e7b24eef3e5cb5d1e0400101650128e9. pdf [Consultado el 9 de mayo de 2017].

39. Lorenzo-Seva U, Ferrando PJ. FACTOR: A computer program to fit the exploratory factor analysis model. Behav Res Methods 2006; 38 (1): 88-91.

40. Muthén L, Muthén B. Mplus User's Guide (7.a ed.). Los
Angeles, USA: Muthén \& Muthén. 2012.

41. Schumacher R, Lomax R. A Beginner's Guide to Structural Equation Modeling. New Jersey: Lawrence Erlbaum Associates, Publishers. 1996.

42. Browne M, Cudeck R. Alternative ways of assessing model fit. En: Bollen K, Long J, Testing Structural Equation Models. Beverly Hills, USA: Sage.1993. p. 136-62.

43. Trizano-Hermosilla I, Alvarado JM. Best Alternatives to Cronbach's Alpha Reliability in Realistic Conditions: Congeneric and Asymmetrical Measurements. Front Psychol 2016; 7: 769.

44. McDonald R. Test Theory: a unified treatment. Mahwah, NJ: Lawrence Erlbaum Associates. 1999.

45. Sijtsma K. On the use, the misuse, and the very limited usefulness of Cronbach's alpha. Psychometrika. 2009; 74(1): 107-20.

46. Torres GV, Reis LA, Reis LA, Fernandes MH. Qualidade de vida e fatores associados em idosos dependentes em uma cidade do interior do Nordeste. J Bras Psiquiatr 2009; 58 (1): 39-44.

47. da Silva MJ, Victor JF, Mota FRN, Soares ES, Leite B MB, Oliveira ET. Analysis of psychometric properties of family APGAR with elderly in northeast Brazil. Esc Anna Nery 2014; 18 (3): 527-32.

48. Zicavo N, Palma C, Garrido G. Adaptación y validación del Faces-20-ESP: Re-conociendo el funcionamiento familiar en Chillán, Chile. Rev Latinoam Cienc Soc Niñez Juv 2012; 10 (1): 219-34.

49. Zegers B, Larraín ME, Polaino-Lorente A, Trapp A, Diez I. Validez y confiabilidad de la versión española de la escala de Cohesión y Adaptabilidad Familiar (CAF) de Olson, Russell \& Sprenkle para el diagnóstico del funcionamiento familiar en la población chilena. Rev Chil Neuro-psiquiatr 2003; 41 (1): 39-54.

50. Puschel K, Repetto P, Solar MO, Soto G, González K. Diseño y validación del instrumento SALUFAM: un instrumento de valoración de la salud familiar con alto valor predictivo clínico para la atención primaria chilena. Rev Med Chile 2012; 140 (4): 417-25.

51. Ferrandi PJ, Lorenza-Seva U. Assessing score determina$\mathrm{cy}$, measurement quality and closeness to unidimensionality in exploratory item factor analysis. Educ Psychol Meas 2018; 78 (5): 762-80. 